\title{
Dual-mode optical microscope based on single-pixel imaging
}

\author{
A. D. Rodríguez ${ }^{\mathrm{a}, *}$, P. Clemente $^{\mathrm{b}}$, E. Tajahuerce ${ }^{\mathrm{a}}$, J. Lancis $^{\mathrm{a}}$ \\ ${ }^{a}$ Institut de Noves Tecnologies de la Imatge (INIT), Universitat Jaume I, Castelló 12071, \\ Spain \\ ${ }^{b}$ Servei Central d'Instrumentació Científica (SCIC), Universitat Jaume I, Castelló 12071, \\ Spain
}

\begin{abstract}
We demonstrate an inverted microscope that can image specimens in both reflection and transmission modes simultaneously with a single light source. The microscope utilizes a digital micromirror device (DMD) for patterned illumination altogether with two single-pixel photosensors for efficient light detection. The system, a scan-less device with no moving parts, works by sequential projection of a set of binary intensity patterns onto the sample that are codified onto a modified commercial DMD. Data to be displayed are geometrically transformed before written into a memory cell to cancel optical artifacts coming from the diamond-like shaped structure of the micromirror array. The 24-bit color depth of the display is fully exploited to increase the frame rate by a factor of 24 , which makes the technique practicable for real samples. Our commercial DMDbased LED-illumination is cost effective and can be easily coupled as an add-on module for already existing inverted microscopes. The reflection and transmission information provided by our dual microscope complement each other and can be useful for imaging non-uniform samples and to prevent self-shadowing effects.
\end{abstract}

Keywords: Microscopy, Reflection, Transmission, Resolution, Computational imaging, Spatial Light Modulators

\section{Introduction}

Optical microscopy techniques have become essential tools in basic and applied research. For instance, in the biological realm, fluorescence microscopy is the method of choice for imaging proteins and their nano-scale dynamic orga5 nization. Despite impressive improvements in optical resolution during the last decades [1, 2, 3], there is still room to improve a range of other features such as the trade-off between the field of view and the depth of field [4, 5], 3D imaging

\footnotetext{
${ }^{*}$ Corresponding author
} 
of specimens [6], or the design of cost-effective, light-weight platforms that can operate in resource-limited settings for global health challenges [7, 8], among others.

Structured illumination microscopy (SIM) stands out among the different optical microscopy techniques because it allows to retrieve spatial frequency information inaccessible with standard uniform illumination schemes [9, 10]. It is based on a standard wide-field optical microscope where the specimen is illumi-

15 nated with a set of known sinusoidal patterns with different phase shifts. The object is reconstructed from multiple acquisitions by using dedicated algorithms. Additionally, this fringe-projection method is attractive because of its intrinsic optical sectioning capability that enables whole-field optically sectioned images [1], 12 .

${ }_{20}$ In the conventional implementation of a SIM, a focal plane array detector detects variations in light intensities scattered by the sample with spatial resolution. Other structured illumination approaches have also been implemented for microscopy during the past few years. The single-pixel imaging (SPI) scheme [13. has gained considerable attention as a very effective sensing mechanism and

25 has triggered diverse applications where conventional cameras equipped with millions of pixels fail to give an adequate response, including optical microscopy 14. As a matter of fact, SPI allows imaging at previously undeveloped spectral bands [15, 16], at the photon-counting regime [17, in presence of strong turbulence or scattering [18, 19, and also through multimode optical fibers [20, 21].

30 The combination of wide-field structured illumination altogether with a bucket detector has made hyperspectral imaging across the visible spectrum possible in a fluorescence microscope [22]. Also, a prototype microscope system based on SPI to image simultaneously in the visible and the short-wave infrared has been recently demonstrated [23].

35 In SPI the problem of spatial resolution is shifted away from the sensor to a set of a microstructured spatial masks that are codified onto a programmable spatial light modulator. The masks are optically projected onto the sample through the microscope objective and the whole intensity is collected onto a bucket (single-pixel) sensor. Measurements are sequential by changing of the

40 spatial mask. If many different masks are used, their shapes and the intensity signals are combined to retrieve the sample. This generalized mask scanning offers several advantages, like signal-to-noise ratio enhancement and the possibility to reduce the acquisition time through compressive sensing, over the traditional raster scan used in confocal microscopy where a single bright pixel 4 is scanned to build up the image [24, 25].

The nature of SPI enforces a reciprocal relationship between the frame rate and image size as the time required to capture an image scales with the number of pixels in the image. Two different approaches can be employed to deal with this issue. On the one hand, given some reasonable assumptions about the

50 sparsity of the signal, compressive sensing dramatically reduces the number of measurements well below the number of pixels of the sample [26, 27]. What is remarkable here is that with the only "a priori" knowledge about the sparsity of the sample it is possible to get rid off the measurement of the full-length signal, 
so it saves time. More recently, adaptive sensing has been introduced as a way to circumvent the computational complexity in convex optimization or greedy algorithms used in compressive sensing $23,28,29$. On the other hand, SPI usually relies on the use of fast spatial light modulators such as the digital micromirror device (DMD) to codify the projecting masks. DMDs permit highly flexible codification of binary masks at frame rates above $20 \mathrm{kHz}$. Extensive application

60 of the DMD to microscopy has been reported in the past few years including conventional SIM microscopy with fringe projection [30, super-resolution and optical sectioning microscopy [31, 32 and the programmable array microscope [33, 34, 35. Interestingly, fast DMD and pattern illumination is at the core of optogenetics, a tool for noninvasive activation and silencing of neurons and 65 muscles 36, 37.

Along the same lines than SPI microscopy, here we demonstrate a dual-mode microscope that can image specimens in reflection and transmission modes simultaneously. Many specimens, such as biological samples, are weak reflectors but produce transmission images with good contrast. Inversely, other samples 70 are very dense and provide poor transmission images or generate self-shadowing effects. Conventional optical microscope designs make the simultaneous collection of transmitted and reflected light inefficient, restrictive, or even impossible. In general they need different light sources for transmission and reflection, thereby preventing that both images be simultaneously measured in a single 75 sensor. Alternatively they can work with several digital cameras, but then a careful calibration and geometric adjustment of both sensors is necessary. The usefulness of dual-mode microscopy for histopathology studies of skin tissue has been recently reported based on a lensless holographic setup [38, 39] and near-field scanning optical microscopy [40].

so Here, we demonstrate that the SPI architecture is particularly well-fitted for this dual operation recording both reflection and transmission information simultaneously with a single light source and a simple light sensor configuration constituted by two single-pixel detectors. The field of view, the optical resolution, and the focused plane are determined by the light projection sys-

85 tem. Therefore, the reflection and transmission images obtained by each light detector are automatically adjusted geometrically with no need of calibration procedures. Furthermore both images are focused unequivocally to the same plane of the sample. In summary, in our microscope, reflection information complements the transmission one very efficiently, which can be very useful for imaging non-uniform samples and to prevent self-shadowing effects.

For the practical implementation, instead of using a high-end DMD as previous single-pixel microscopy techniques, we use of an off-the-shelf DMD from a cost-effective digital light projector. These devices utilize an offset diamond pixel layout which generates geometrical artifacts. In addition to prove the dual ${ }_{5}$ mode operation, in this work we demonstrate an algorithm to precisely allocate pixels in memory to deal with this problem. 


\section{Dual-mode single-pixel imaging}

In a dual-mode SPI microscope, the DMD plane is relayed by a convenient optics onto the sample plane and the forward and backscattered light components are simultaneously collected onto dual single-pixel photodetectors located at the transmission and reflection ports of the microscope, respectively [see Fig. 1. A sequence of $M$ sampling patterns is codified onto the DMD so that the irradiance striking the photodetector at the transmission port in the $i$ th timestep is

$$
Y_{i}=\left\langle\Psi_{i}(\vec{x}), T(\vec{x})\right\rangle,
$$

where $T(\vec{x})$ is the transmittance distribution of the sample, $\Psi_{i}(\vec{x})$ denotes the $i$ th measurement pattern, and $\left\langle\Psi_{i}(\vec{x}), T(\vec{x})\right\rangle$ represents the inner product between both functions. The state of the sampling projector $\Psi_{i}(\vec{x})$ is changed from one timestep to the next to implement a full set of measurements

$$
\mathbf{Y}=\mathbf{S T}
$$

where $\mathbf{S}$ is a sensing matrix whose $i$ th row is a one-dimensional reshaping of the $i$ th sampling mask $\Psi_{i}(\vec{x})$, and $\mathbf{T}$ and $\mathbf{Y}$ are $N$-dimensional vectors representing a one-dimensional reshaping of the unknown transmittance distribution and the result of the measurements at the transmission port, respectively.

Equivalently, the measurements at the reflection port are concisely represented by the series of linear equations

$$
\mathbf{Z}=\mathbf{S R},
$$

where $\mathbf{R}$ and $\mathbf{Z}$ are $N$-dimensional vectors representing a one-dimensional reshaping of the unknown reflectance distribution and the result of the measurements at the reflection port, respectively,

The problem of the measurement can be stated as: given the result of the measurements $\mathbf{Y}$ and $\mathbf{Z}$ derive the set of values $\mathbf{T}$ and $\mathbf{R}$ that best represent the transmittance and reflectance distributions $T(\vec{x})$ and $R(\vec{x})$ of the sample.

In the easiest implementation of the retrieval algorithm, which is possible for well-conditioned measurements systems, a number of measurements $M$ equal to the number of pixels of the sample is required and both the transmission and the reflection images are retrieved through the inverse matrix as

$$
\mathbf{T}=S^{-1} \mathbf{Y} \quad, \quad \mathbf{R}=S^{-1} \mathbf{Z} .
$$

Concerning the measurement patterns, various matrices can be employed. For instance, raster-scan style masks stem from the well-known raster-scan technique in which spatial pixels are measured sequentially. Random matrices can also be used in which each mask has a random distribution of binary values. On the other hand the Hadamard S matrix provides a convenient codification framework because binary nonnegative elements can easily be displayed onto the DMD [41]. The Hadamard S matrix is defined through the equation 


$$
\mathbf{S}_{N}=\frac{1}{2}\left(1-\mathbf{H}_{N}\right)
$$

where

$$
\mathbf{H}_{N} \mathbf{H}_{N}^{\tau}=N \mathbf{I}_{\mathbf{N}}
$$

with $\mathbf{H}_{N}$ the Hadamard matrix of order $N, \mathbf{H}_{N}^{\tau}$ the transposed matrix, and $\mathbf{I}_{N}$ the $N \times N$ identity matrix. Also the above choice provides minimum variance least-squares estimation of the unknown variables. Further, Hadamard measurements obtained by subtracting $S$-matrix measurements from complementary patterns produce a better result in a similar way to the results achieved through balanced detection [42, 43].

\subsection{Compressive sensing retrieval}

Although there are some benefits of using SPI in optical microscopy, it increases image acquisition time over conventional imaging using focal plane array detectors due to the fact that measurements must be taken sequentially, rather than in parallel. To boost up the image acquisition, we take advantage of the ground-breaking theory of compressive sensing (CS), which makes it possible to recover an $N$-pixels image from $M<N$ measurements [24, 25]. In CS, one takes samples of the $\mathrm{N}$-dimensional transmission vector $\mathbf{T}$ using a matrix $M \times N$ matrix $S^{\prime}$, obtaining undersampled measurements $\mathbf{Y}^{\prime}=S^{\prime} \mathbf{T}$. Along this section, and with no lack of generality, we will refer to the transmission signal, where an identical relation holds for the reflection mode.

Although the above equation defines an underdetermined linear relation with a number of equations lower than the number of variables $(M<N)$, the restricted isometry property guarantees that the right signal can still be reconstructed if we simply assume it has a low information content compared with its physical dimensions. In mathematical terms, we assume that the signal is sparse in some basis representation. The problem then becomes how to sample properly with a measurement matrix satisfying certain conditions. For random measurement matrices with independent standard Gaussian entries $S^{\prime}$, it is known that convex optimization $\min \|\mathbf{T}\|_{1}$ subject to $\mathbf{Y}^{\prime}=S^{\prime} \mathbf{T}$ typically finds the sparsest solution. In the above equation, $\|\cdot\|_{1}$ denotes the $l_{1}$ norm. Yet, ${ }_{160}$ there are different methods to retrieve the image such as basis pursuit methods and greedy pursuit methods.

Also, it has been shown that deterministic measurement ensembles exhibit the same behavior than Gaussian matrices via $l_{1}$ minimization. Analytically known matrices avoid the need to store the entire matrix as the entries can be computed on the fly and permit to use recovery algorithms with lower complexities. Note that operation at video rates is of paramount significance in applications of SPI in optical microscopy [21,27]. Further, partial Hadamard ensembles where the matrix $S^{\prime}$ is formed by uniform random selection of $M$ rows of the Hadamard transform matrix allow to acquire biologically relevant 
samples [20]. In this case, it is essential to enforce the sparsity of the reconstruction signal in some representation with a high level of dissimilarity with the elements of the measurement matrix.

Because measurements are noisy, it is better to relax the minimization constraints to

$$
\min \|\mathbf{T}\|_{1} \quad \text { subject to }\left\|\mathbf{Y}^{\prime}=S^{\prime} \mathbf{T}\right\|_{2}<\epsilon,
$$

and ask that the fit holds to the noise level $\epsilon$. The full performance analysis of the compressive sensing methodology in terms of the dynamic range of the sensor, the bit depth of the A/D converter, and the amount of noise was conducted in Reference [13].

In our study, we utilized the $l_{1}$-magic solver [44] to reconstruct the images with partial Hadamard ensemble to form the measurement matrix. Yet, as the power spectrum of most biological images is generally concentrated at low frequencies, we employed a sampling strategy that favours this frequency range. While this is true, biological samples also have fine details that could be lost due to this sampling strategy. However, in this work, for low frequencies we refer to low Hadamard frequencies, which also include high frequencies in Fourier space.

\section{Experimental set-up}

In this section we describe how to setup the dual-mode single-pixel microscope by attaching a low-cost commercial DMD and two photodetectors to an inverted microscope. In order to have a low-cost DMD with standard video in-

190 put control, we remove the optics and the illumination system of an off-the-shelf digital light projector (DLP). We use the illumination system of the commercial microscope and we project the display through the epifluorescence port of the microscope and transfer a demagnified image to the sample plane [Fig. 1]. The inverted microscope used in the experiment is a Nikon Eclipse T $i$ - $\mathrm{U}$. The system can be divided in two parts: the illumination system and the collection system. The illumination system is composed by a mercury lamp (Nikon, Intensilight C-HGFI), a DMD (DLP LightCrafter ${ }^{\mathrm{TM}}$ EVM 0.3 WVGA), two relay lenses $(\oslash=2$ " achromatic doublets, with focal lengths 100mm (L1) and 200mm (L2)) producing a $2 \mathrm{X}$ magnification, a tube lens and the projection objective (Nikon 200 LU Plan 20X/0.40 WD 13). The DMD is illuminated with a collimated light beam. An intermediate image of the DMD is projected onto the focal plane of the tube lens by the relay lenses and then demagnified by the projection objective onto the sample plane. The two collection systems for reflection and transmission are composed by a condenser lens and a photomultiplier tube PMT (PMM01, Thorlabs Inc.). The reflection PMT is placed in a lateral port and the transmission PMT is placed above the stage of the microscope.

\subsection{Pattern codification on diamond shaped DMDs}

The direct implementation of an $N \times N$ image onto the diamond pixel layout differs from the implementation onto the conventional orthogonal pixel layout. 


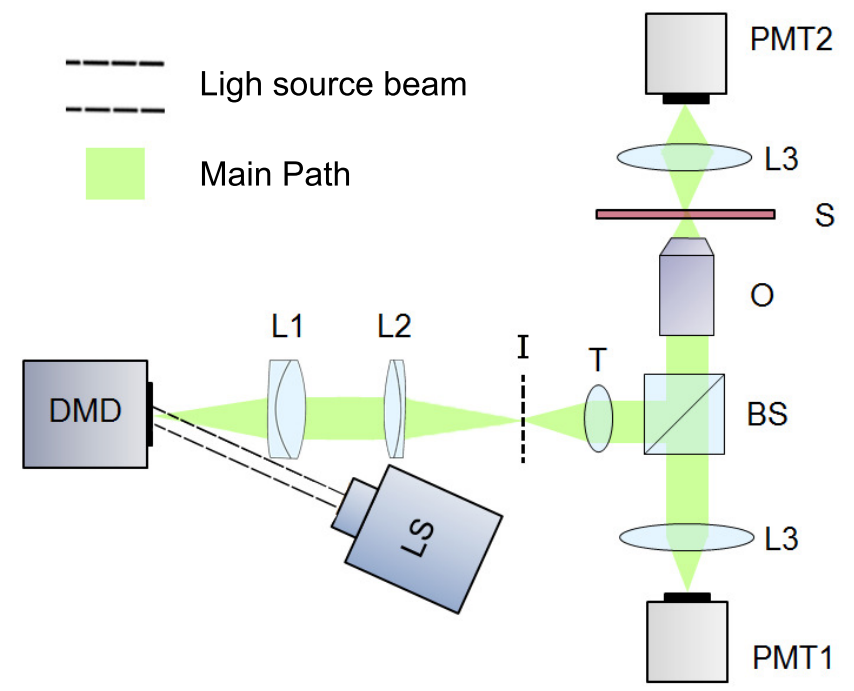

Figure 1: Experimental set-up. (LS) Light Source. (DMD) Digital micromirror device. (L1, L2) Relay lenses. (I) Intermediate image of the DMD. (T) Tube lens. (BS) Beam splitter. (S) Sample plane. (L3) Condenser lenses. (O) Projection objective. Reflection and transmission photomultiplier tubes (PMT1 and PMT2 respectively).

In an orthogonal layout the rows and the columns are straight lines, so the aspect ratio is preserved. However in the diamond layout meanwhile the rows are straight lines, the columns are zigzag lines. Thereby the direct implementation changes the original aspect ratio of the image. Moreover, straight edges are transformed on zigzag edges. Both consequences are shown in Fig. 2
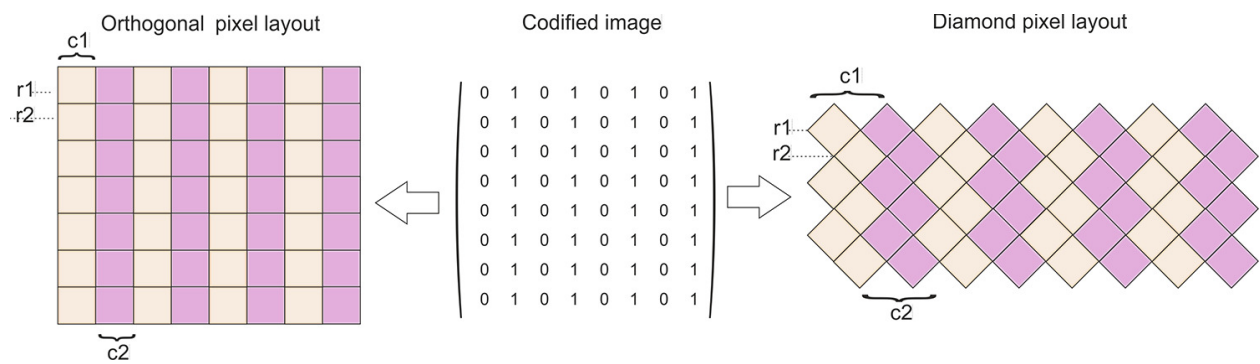

Figure 2: Graphical representation of the different coordinate system for orthogonal pixel layout (left) and diamond pixel layout (right).

To appreciate the effects due to miscoding of the patterns in image retrieval within the framework of SPI, we reconstructed the transmission mode of a negative USAF test chart (USAF $19512 \times 2$ ) that was used as a sample in the optical setup of Fig. 1. The number of projected test patterns (Hadamard patterns) was 4096 , with a resolution of $64 \times 64$ pixels, using no compression techniques. The result is shown in Figure 3 . 


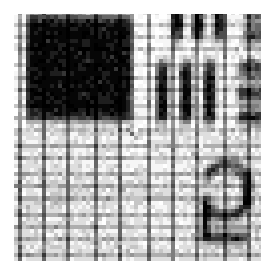

Figure 3: Experimental image reconstructed without pattern predistortion $(64 \times 64$ pixels $)$ for the transmission mode. Geometrical artifacts (phantom grid) due to the diamond layout are clearly noticeable.

To avoid these artifacts, before sending an image to the DMD, the information must be codified in a different way. The aspect ratio could be preserved pre-distorting the original image but this operation do not correct the zigzag edges artifact. In this work a subtler coding is presented that preserves both the aspect ratio and edges of the original image. An example of the operation of our coding algorithm is presented in Fig. 4(a) and (b) for the case of a $2 \times 2$ image. Without correction, information codified in a $2 \times 2$ array provides a distorted image in the diamond layout as is shown in Fig. 4(a). Our algorithm codifies the information in an array in such way that original image appears rotated but preserves the aspect ratio and straight edges as shown in Fig. 4(b).

The array transformation produced by the algorithm is shown in Fig. 4(c). In general, the algorithm transforms an $N \times N$ image (where $N$ is even) into a $(2 N-1) \times N$ array, following the next steps:

1. Diagonals are extracted from the initial image in the order shown in Fig. 4(c).

2. Each row of the $(2 N-1) \times N$ image is built by zero-padding each diagonal. The order of the rows matches the order of the extracted diagonals. If the row is even, it is padded to left and right of the diagonal with the same number of zeros. If the row is odd, an additional zero is needed to the left.
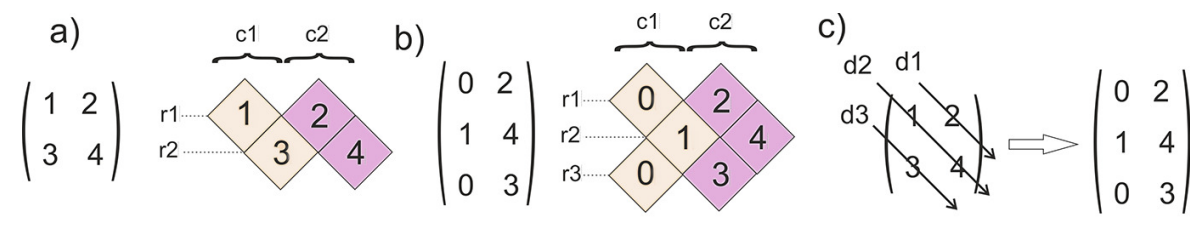

Figure 4: Graphical representation of the matrix transformation applied to the projected patterns to avoid artifacts.

In this way, by means of this previous transformation, the coding of a square image is displayed as a square image rotated 45 degrees, preserving the original aspect ratio and edges. 


\subsection{Temporal multiplexing of binary patterns}

Commercial DMDs from video projectors are slower than scientific DMDs created specifically for scientific applications, as they work only at video rates $(60-120 \mathrm{~Hz})$. To improve speed performance, we take advantage of the codification procedure used by the DLP for color video projection. In the standard video projection mode, the light changes sequentially to blue, red and green colors. For each color, the display codifies 8 binary patterns. An improvement in speed can be done by codifying 24 binary patterns within a single 24-bit depth image. This technique allows an improvement in speed rate of 24 , going from $60-120 \mathrm{~Hz}$ to $1440-2880 \mathrm{~Hz}$. A schematic overview of the codification is shown in Fig. 5. The DMD was fully controlled by software designed by our group in Labview $^{\mathrm{TM}}$. The image processing was performed using Matlab ${ }^{\mathrm{TM}}$.

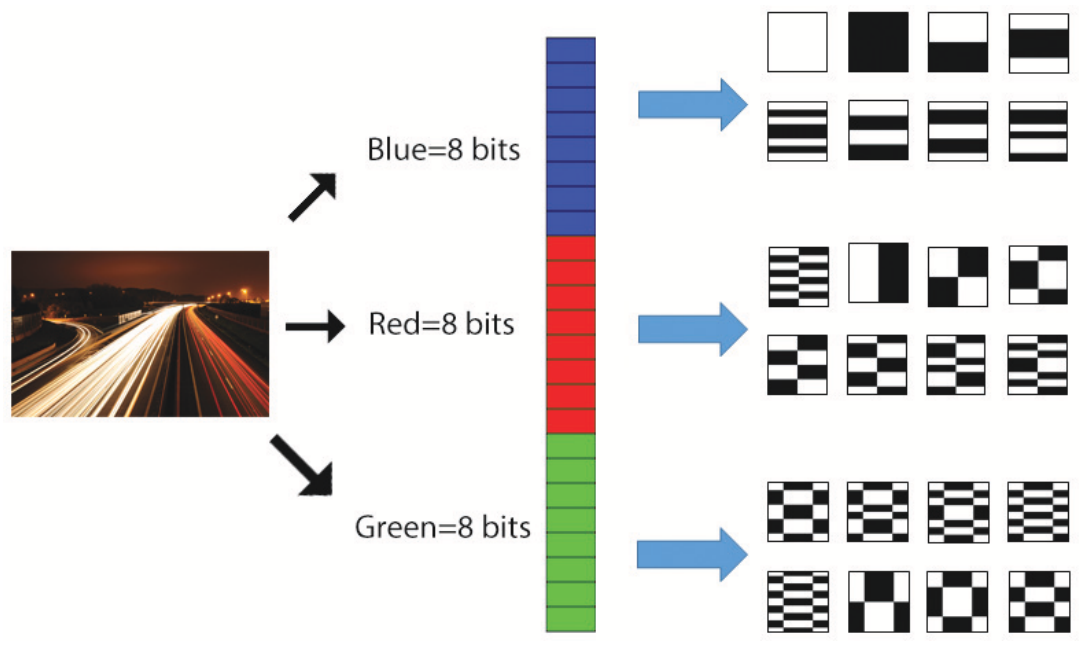

Figure 5: Codification of a 24-bit image with 24 Hadamard patterns of 1-bit depth. This technique allows an improvement in speed rate of 24 , going from $60-120 \mathrm{~Hz}$ to $1440-2880 \mathrm{~Hz}$.

In Fig. 6 we plot the temporal response of the transmission photomultiplayer tube for a single 24-bit image. The patterns projected and the object under measure were the same as in the experiment seen in Fig. 3. Each value for $Y_{i}$ is obtained by averaging the values within the ranges marked by a red line. Because the number of patterns exceeds the projector's internal memory we have to send the patterns through commercial video stream. Through this channel, the video projector repeats each image 6 times (for visual purposes). The maximum speed rate reached was $1440 / 6=240 \mathrm{~Hz}$, allowing us to capture a $64 \times 64$ image (with no compression techniques) in 17 seconds.

\section{Results}

With the aim of analyzing the optical resolution in both transmission and reflection modes, a negative USAF test chart (USAF $19512 \times 2$ ) is employed as 


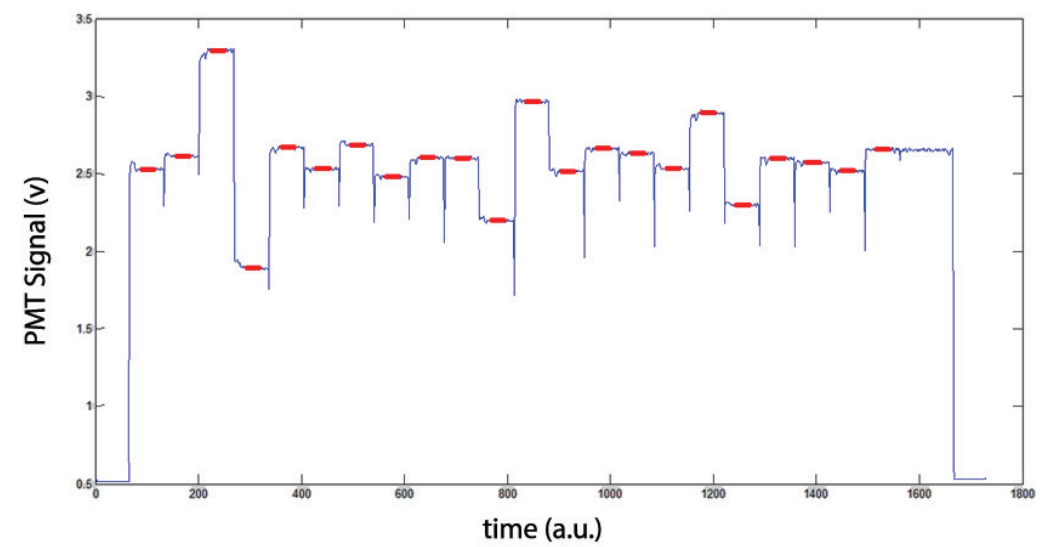

Figure 6: Photocurrent values for a single 24-bit frame. In the graphic the different values for each 1-bit depth pattern codified in the 24-bit image are shown in red color. In this case, the first and last bit are use as reference.

an object in the setup shown in Fig. 1. The microscope objective is a 20X with $0.40 \mathrm{NA}$. Only the smallest groups of the USAF test, 6 and 7 , were used to bound the resolution of the system. The scales and dimensions of the bars are given

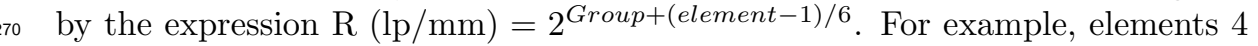
and 5 of group 7 have a resolution of $181.0 \mathrm{lp} / \mathrm{mm}$ and $203.2 \mathrm{lp} / \mathrm{mm}$, equivalent to a feature size of $2.76 \mu \mathrm{m}$ and $2.46 \mu \mathrm{m}$ respectively. The images were obtained as follows. The patterns of the basis were projected onto the object plane by $\mathrm{L} 1$ and $\mathrm{O}$, then the intensity transmitted and reflected by the object for each 275 pattern were captured, simultaneously by the two PMTs. The experimental results are shown in Fig. 7. To boost the sensing stage, compressive sensing algorithms were used. Binning is performed by codifying each pixel of the Hadamard patterns with a group of $4 \mathrm{x} 4$ micromirrors in the DMD. Pictures (a) and (b) show transmission and reflection images, respectively, by using only $28025 \%$ of the total number of patterns $(M=1,012)$ for a resolution of $64 \times 64$ pixels while (c) and (d) have a $50 \%$ compression $(M=2,046)$ for the same resolution. Pictures (e) and (f) were obtained by projecting the total number of patterns, without compression $(M=4,092)$. The results show that the resolution obtained by transmission and reflection are almost identical $(2.46 \mu \mathrm{m}$ 285 for the images with no compression) and that reducing the projected patterns to $50 \%$ does not significantly affect resolution $(2.76 \mu \mathrm{m})$ but allows measuring twice as fast. These two resolutions values are obtained from the last resolvable element of group 7 (5 and 4 respectively). In our experimental set-up, the PMT can detect light signals at frequencies as high as $20 \mathrm{kHz}$. However, the acquisition frequency is limited to $60 \mathrm{~Hz}$ by the graphic card and, therefore, to $1440 \mathrm{~Hz}$ by the frame rate of the commercial grade DMD.

The quality of the compressed images was tested using the standard peak signal-to-noise ratio, $P S N R=10 \log \left(I_{\max }^{2} / \mathrm{MSE}\right)$, where $I_{\max }$ is the maximum 
pixel intensity value of the reference image and $\mathrm{MSE}=\left(\frac{1}{N}\right)\left\|\mathbf{I}_{r e f}^{i n}-\mathbf{I}_{r e c}^{i n}\right\|^{2}$, where 295 $\mathbf{I}_{r e c}^{i n}$ is the undersampled image and $\mathbf{I}_{r e f}^{i n}$ is the image recovered from the whole measurement. The result of this analysis for the case of the reflection mode is shown in Fig 8. The figure shows a plot of the PSNR together with the reconstructed image for different compression ratios.

(a)
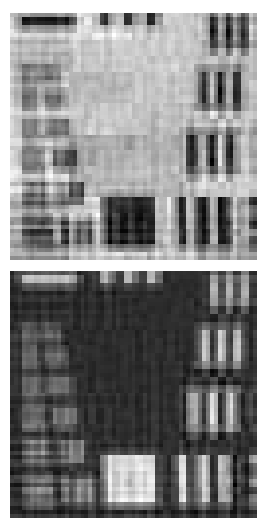

(c)
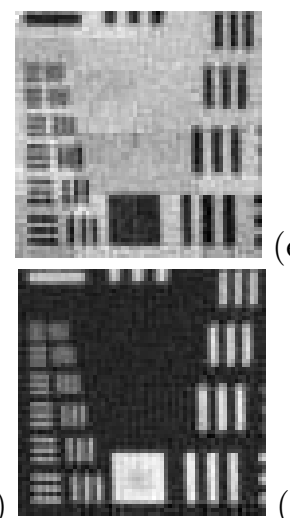

(e)
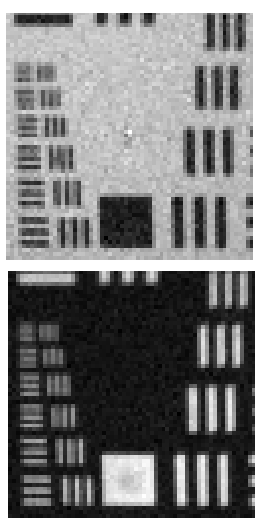

Figure 7: Experimental images of the USAF test (groups 6 and 7) obtained with the dualmode single-pixel microscope in Fig. 1] using compressive sensing algorithms with a different compression ratio. Pictures (a) and (b) show transmission and reflection images, respectively, by using only $25 \%$ of the total number of patterns for this resolution $(64 \times 64$ pixels $)$ and $50 \%$ (c-d) of the whole number of patterns. (e-f) Transmission and reflection images with no compression. In all cases, the size of the image was $64 \times 64$ pixels and the field of view $192 \mu m \times 192 \mu m$.

As a proof of concept, we have also tested the capability of the dual-mode SPI microscope to image biological samples with different reflective and transmissive imaging profiles. The results can be seen in Fig. 9. The first row show images of an Anaphothrips obscurus in both reflection (a) and transmission (b) modes taken simultaneously. The images have a resolution of $64 \times 64$ pixels $(M=$ 4,096 , binning $=4 \times 4$ micromirrors) and were obtained with a $20 \mathrm{X}$ microscope objective. In this case the transmission image shows only the silhouette of the insect because of its low transparency. The second row show images of a Zeuzera pyrina wing taken with a single-pixel camera in reflection (c) and transmission (d) modes, respectively. Both images have a resolution of $128 \times 128$ pixeles ( $M=16,384$, binning $=2 \times 2$ micromirrors). In this case, the transmission 310 image shows structures of the other side of the wing (hairs), hidden in the reflection images. The images of both modes are focused to the same plane of the sample and are automatically adjusted geometrically. This can be an advantage for biological structural studies. Image (e) show the same area of the sample taken in reflection with a CMOS sensor located at the output port of the microscope. This time the resolution of the image is $200 \times 200$ pixeles. 


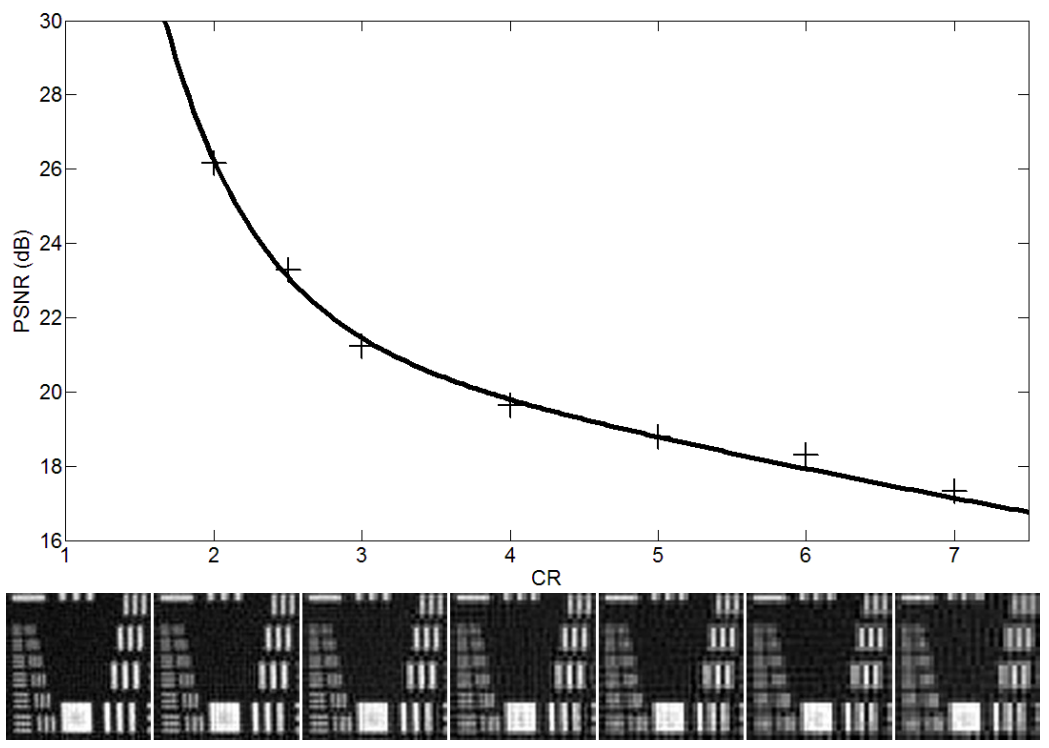

Figure 8: (Top) Plot of the standard peak signal-to-noise ratio (PSNR) in dB as a function of the compression ratio $(C R=N / M)$ to show the quality of the recovered images for the case of the reflection mode. (Bottom) Images reconstructed with the different compression ratios associated with the PSNR plotted in the top figure with increasing CR from left to right.

(a)

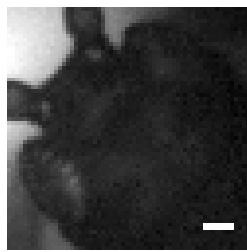

(c)

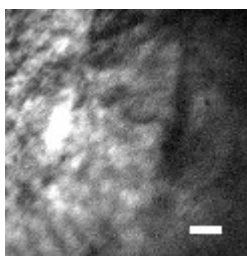

(b)

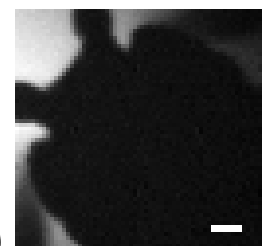

(d)

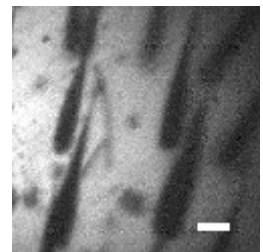

(e)

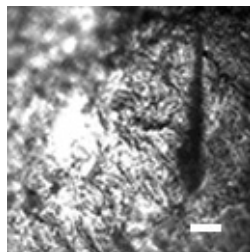

Figure 9: Experimental results. Anaphothrips obscurus in reflection (a) and transmission (b) modes obtained with the optical setup in Fig. 1 using a $20 \mathrm{X}$ objective. The images have $64 \times 64$ px. Images of a Zeuzera pyrina wing in reflection (c) and transmission (d) modes. In this case the images have a resolution of $128 \times 128 \mathrm{px}$. (e) Image in reflection taken with a CMOS sensor located at the output port of the microscope. $(200 \times 200 \mathrm{px})$. Scale bar: $25 \mu \mathrm{m}$. 


\section{Conclusion}

In conclusion, we have developed a dual-mode optical microscope by attaching a commercial DMD and two single-pixel detectors to an inverted microscope. We have designed an algorithm to modify the shape of the projected patterns

320 called diamond pixel architecture. We have demonstrated that our system is able to retrieve images of reflection and transmission modes with the same field of view and from the same plane of the sample, revealing structures not visible easily by conventional microscopy techniques. This is done with no need of are controlled exclusively by the properties of the light patterns generated by the optical projection system. By recording transmitted and reflected information loss of data by self-shadowing is minimized which can be advantageous in non-uniform samples containing transparent and opaque regions. Both imaging 330 modes benefit from the advantages present in single-pixel imaging as low-light sensitivity as well as multidimensional capability through the use of specialized sensors. Single-pixel imaging can also be advantageous in the presence of optical aberrations and optical imperfections in between the sample and the sensor. In our case, this is a clear advantage as the mode of transmission has to go through the entire sample before reaching the sensor. Our system benefits also from the advantages of compressive sensing, reducing the measure time by $50 \%$ without strongly affecting the resolution of the system. This fact allows us to configure the system with a compromise between acquisition speed and resolution. Additionally, the simplicity of the detection system will allow us to

340 record different spectral channels or polarization states in each imaging mode very easily, increasing the versatility of the system.

\section{Acknowledgments}

This work was supported by MINECO trough projects FIS2013-40666-P, the Generalitat Valenciana PROMETEO/2012/021, ISIC/2012/013, and by the

345 Universitat Jaume I P1-1B2012-55. A. D. Rodríguez acknowledges grant PREDOC/2012/41 from Universitat Jaume I. Thanks also to Dr. Tatiana Pina and Dr. Josep Jaques from Universitat Jaume I for providing us the biological samples.

\section{References}

[1] S. W. Hell, J. Wichmann, Breaking the diffraction resolution limit by stimulated emission: stimulated-emission-depletion fluorescence microscopy., Optics letters 19 (11) (1994) 780-782. doi:10.1364/0L.19.000780.

[2] E. Betzig, G. H. Patterson, R. Sougrat, O. W. Lindwasser, S. Olenych, J. S. Bonifacino, M. W. Davidson, J. Lippincott-Schwartz, H. F. Hess, 
(New York, N.Y.) 313 (5793) (2006) 1642-1645. doi:10.1126/science. 1127344

[3] W. E. W. E. Moerner, Single-Molecule Spectroscopy, Imaging, and Photocontrol: Foundations for Super-Resolution Microscopy (Nobel Lecture)., Angewandte Chemie (International ed. in English) 54 (28) (2015) 8067-93. doi:10.1002/anie.201501949. URL http://www.ncbi.nlm.nih.gov/pubmed/26088273

[4] G. Zheng, R. Horstmeyer, C. Yang, Wide-field, high-resolution Fourier ptychographic microscopy. Nature photonics 7 (9) (2013) 739-745. doi: 10.1038/nphoton.2013.187.

URL http://dx.doi.org/10.1038/nphoton.2013.187

[5] L. Tian, L. Waller, 3D intensity and phase imaging from light field measurements in an LED array microscope, Optica 2 (2) (2015) 104-111. doi:10.1364/OPTICA.2.000104.

[6] R. Prevedel, Y.-G. Yoon, M. Hoffmann, N. Pak, G. Wetzstein, S. Kato, T. Schrödel, R. Raskar, M. Zimmer, E. S. Boyden, A. Vaziri, Simultaneous whole-animal 3D imaging of neuronal activity using light-field microscopy.,

n Nature methods 11 (7) (2014) 727-30. arXiv:1401.5333, doi:10.1038/ nmeth.2964.

375 URL http://www.ncbi.nlm.nih.gov/pubmed/24836920

[ [7] A. Ozcan, Mobile phones democratize and cultivate next-generation imaging, diagnostics and measurement tools., Lab on a chip (2014) 31873194doi:10.1039/c4lc00010b.

URL http://www.ncbi.nlm.nih.gov/pubmed/24647550

[8] A. F. Coskun, A. Ozcan, Computational imaging, sensing and diagnostics for global health applications, Current Opinion in Biotechnology 25 (2014) 8-16. doi:10.1016/j.copbio.2013.08.008.

URL http://dx.doi.org/10.1016/j.copbio.2013.08.008

[9] M. G. Gustafsson, Surpassing the lateral resolution limit by a factor of two using structured illumination microscopy., Journal of microscopy $198(\mathrm{Pt}$ 2) (2000) 82-87. doi:10.1046/j.1365-2818.2000.00710.x.

[10] M. G. L. Gustafsson, Nonlinear structured-illumination microscopy: widefield fluorescence imaging with theoretically unlimited resolution., Proceedings of the National Academy of Sciences of the United States of America 102 (37) (2005) 13081-13086. doi:10.1073/pnas.0406877102.

[11] J. R. Allen, S. T. Ross, M. W. Davidson, Structured illumination microscopy for superresolution. Chemphyschem : a European journal of

n chemical physics and physical chemistry 15 (4) (2014) 566-76. doi: 10.1002/cphc.201301086.

395 URL http://www.ncbi.nlm.nih.gov/pubmed/24497374 
[12] M. Saxena, G. Eluru, S. S. Gorthi, Structured illumination microscopy Advances in Optics and Photonics 7 (2015) 241-275. arXiv:1303.6810 doi:10.1364/AOP.

http://wWW.opticsinfobase.org/abstract.cfm?URI= aop-5-2-131\$\delimiter"026E30F\$nhttp://arxiv.org/abs/1303. 6810

[13] M. F. Duarte, M. a. Davenport, D. Takhar, J. N. Laska, K. F. Kelly, R. G. Baraniuk, J. Romberg, Single-Pixel Imaging via Compressive Sampling, IEEE Signal Processing Magazine 25 (2) (2008) 14-20.

405 URL http://ieeexplore.ieee.org/lpdocs/epic03/wrapper.htm? arnumber $=4472247$

[14] Y. Wu, P. Ye, I. O. Mirza, G. R. Arce, D. W. Prather, Experimental demonstration of an Optical-Sectioning Compressive Sensing Microscope ( CSM ), Optics express 18 (24) (2010) 24565-24578.

[15] C. M. Watts, D. Shrekenhamer, J. Montoya, G. Lipworth, J. Hunt, T. Sleasman, S. Krishna, D. R. Smith, W. J. Padilla, Terahertz compressive imaging with metamaterial spatial light modulators, Nat. Photon. 8 (2014) 605. doi:10.1038/nphoton.2014.139. URL http://www. nature.com/doif inder/10.1038/nphoton.2014.139

415 [16] J. Greenberg, K. Krishnamurthy, D. Brady, Compressive single-pixel snap[1 shot x-ray diffraction imaging., Optics letters 39 (1) (2014) 111-4. doi: 10.1364/OL.39.000111.

[17] E. van den Berg, E. Candès, G. Chinn, C. Levin, P. D. Olcott, C. SingLong, Single-photon sampling architecture for solid-state imaging sensors., ${ }_{420}$ Proceedings of the National Academy of Sciences of the United States of America 110 (30) (2013) E2752-61. arXiv:arXiv:1209.2262v1, doi:10.1073/pnas.1216318110.

II URL http://www.pubmedcentral.nih.gov/articlerender.fcgi? artid $=3725070 \&$ tool=pmcentrez\&rendertype=abstract

${ }_{425}$ [18] E. Tajahuerce, V. Durán, P. Clemente, E. Irles, F. Soldevila, P. Andrés,

口 J. Lancis, Image transmission through dynamic scattering media by single-pixel photodetection, Optics Express 22 (14) (2014) 16945.

1) URL http://www.opticsinfobase.org/abstract.cfm?URI= oe-22-14-16945

${ }_{430}$ [19] V. Durán, F. Soldevila, E. Irles, P. Clemente, E. Tajahuerce, P. Andrés, J. Lancis, Compressive imaging in scattering media, Optics Express 23 (11) (2015) 14424. doi:10.1364/0E.23.014424 URL http://www.osapublishing.org/viewmedia.cfm?uri= oe-23-11-14424\&seq=0\&html=true 
[23] N. Radwell, K. J. Mitchell, G. GIbson, M. Edgar, R. Bowman, M. J. Padgett, Single-pixel infrared and visible microscope, Optica 1 (5) (2014) 285289.

[24] T. Wilson, Resolution and optical sectioning in the confocal microscope, $\mathrm{J}$ 455 $\mathrm{X}$

URL http://www.ncbi.nlm.nih.gov/pubmed/22004276

[25] A. D. Rodríguez, P. Clemente, E. Irles, E. Tajahuerce, J. Lancis, Resolution analysis in computational imaging with patterned illumination and bucket 460 detection. Optics letters 39 (13) (2014) 3888-91.

URL http://www.ncbi.nlm.nih.gov/pubmed/24978763

[26] E. J. Candès, J. Romberg, T. Tao, Robust uncertainty principles: Exact signal reconstruction from highly incomplete frequency information, 4 140409186, doi:10.1109/TIT.2005.862083.

[27] D. L. Donoho, Compressed sensing IEEE Transactions on Information Theory 52 (4) (2006) 1289-1306. arXiv:1204.4227v1, doi:Doi10.1109/Tit.2006.871582

a URL <GotoISI>://000236714000001\$ \delimiter"026E30F\$nhttp: \&arnumber $=1614066 \&$ isnumber $=33885$

[28] M. A $\beta$ mann, M. Bayer, Compressive adaptive computational ghost imaging. Scientific reports 3 (2013) $1545 . \quad$ arXiv:1304.0243, doi:10.1038/srep01545. 
URL http://www.pubmedcentral.nih.gov/articlerender.fcgi? artid=3607834\&tool=pmcentrez\&rendertype=abstract

[29] M. P. Edgar, G. M. Gibson, R. W. Bowman, B. Sun, N. Radwell, K. J. Mitchell, S. S. Welsh, M. J. Padgett, Simultaneous real-time visible and infrared video with single-pixel detectors, Scientific Reports 5 (2015) 10669. doi:10.1038/srep10669. URL http://www.nature.com/doifinder/10.1038/srep10669

[30] T. Fukano, A. Miyawaki, Whole-field fluorescence microscope with digital micromirror device: imaging of biological samples., Applied optics 42 (19) (2003) 4119-4124. doi:10.1364/A0.42.004119.

[31] W. Dan, Dan; Lei, Ming; Yao, Baoli; Wang, Wen; Winterhalder, Martin; Zumbusch, Andreas; Qi, Yujiao; Xia, Liang; Yan, Shaohui; Yang, Yanlong; Gao, Peng; Ye, Tong; Zhao, DMD-based LED-illumination Superresolution and optical sectioning microscopy, SCIENTIFIC REPORTS 3.

[32] D. Xu, T. Jiang, A. Li, B. Hu, Z. Feng, H. Gong, S. Zeng, Q. Luo, Fast optical sectioning obtained by structured illumination microscopy using a digital mirror device., Journal of biomedical optics 18 (6) (2013) 060503. doi:10.1117/1.JB0.18.6.060503. URL http://www.ncbi.nlm.nih.gov/pubmed/23757041

[33] P. J. Verveer, Q. S. Hanley, P. W. Verbeek, L. J. Van Vliet, T. M. Jovin, Theory of confocal fluorescence imaging in the programmable array microscope (PAM), Journal of Microscopy 189 (3) (1998) 192-198. doi:10.1046/j.1365-2818.1998.00336.x.

[34] Q. S. Hanley, P. J. Verveer, M. J. Gemkow, D. Arndt-Jovin, T. M. Jovin, An optical sectioning programmable array microscope implemented with a digital micromirror device, Journal of Microscopy 196 (3) (1999) 317-331. doi:10.1046/j.1365-2818.1999.00602.x.

[35] F. P. Martial, N. A. Hartell, Programmable illumination and high-speed, multi-wavelength, confocal microscopy using a digital micromirror., PloS one 7 (8) (2012) e43942. doi:10.1371/journal.pone.0043942.

505 URL http://journals.plos.org/plosone/article?id=10.1371/ journal.pone.0043942

[36] S. Sakai, K. Ueno, T. Ishizuka, H. Yawo, Parallel and patterned optogenetic manipulation of neurons in the brain slice using a DMD-based projector Neuroscience Research 75 (1) (2013) 59-64. doi:10.1016/j.neures.2012. 03.009 .

URL http://dx.doi.org/10.1016/j.neures.2012.03.009

[37] E. Papagiakoumou, Optical developments for optogenetics, Biology of the Cell 105 (10) (2013) 443-464. doi:10.1111/boc.201200087. 
[38] M. Lee, O. Yaglidere, A. Ozcan, Field-portable reflection and transmission microscopy based on lensless holography, Biomedical Optics Express 2 (9) (2011) 2721. doi:10.1364/BOE.2.002721.

[39] N. A. Talaikova, A. L. Kalyanov, V. P. Ryabukho, Diffraction phase microscopy with transmission and reflection illumination for refractive index measurements, in: P. Ferraro, S. Grilli, M. Ritsch-Marte, D. Stifter (Eds.), SPIE Optical Metrology, International Society for Optics and Photonics, 2015, p. 95291D. doi:10.1117/12.2181946.

(1) URL http://proceedings.spiedigitallibrary.org/proceeding. aspx?articleid $=2344925$

[40] P.-K. Wei, C.-C. Wei, J.-H. Hsu, W.-S. Fann, Simultaneous reflection and transmission modes near-field scanning optical microscope, Ultramicroscopy 61 (1-4) (1995) 237-239.

a URL http://www.sciencedirect.com/science/article/ B6TW1-3YYV254-17/2/5b72fb980c73b72e2bac8bbae4119f40

[41] N. J. Sloane, M. Harwit, Masks for Hadamard transform optics, and weigh-

530 ing designs., Applied optics 15 (1) (1976) 107-114. doi:10.1364/A0.15. 000107.

[42] W.-K. Yu, X.-F. Liu, X.-R. Yao, C. Wang, Y. Zhai, G.-J. Zhai, Complementary compressive imaging for the telescopic system., Scientific reports 4 (2014) 5834. doi:10.1038/srep05834.

535 URL http://www.ncbi.nlm.nih.gov/pubmed/25060569

[43] W.-K. Yu, X.-R. Yao, X.-F. Liu, L.-Z. Li, G.-J. Zhai, Three-dimensional single-pixel compressive reflectivity imaging based on complementary modulation, Appl. Opt. 54 (3) (2015) 363-367. doi:10.1364/A0.54.000363.

URL http://ao.osa.org/abstract.cfm?URI=ao-54-3-363

${ }_{540}$ [44] E. J. Candes, $l_{1}$-magic.

URL http://users.ece.gatech.edu/justin/l1magic 